June 2008

\title{
Estrogen replacement therapy induces functional asymmetry on an odor memory/discrimination test
}

Richard L. Doty

Mehreen Kisat

Aga Khan University

Isabelle Tourbier

Follow this and additional works at: https://ecommons.aku.edu/

pakistan_fhs_mc_surg_otolaryngol_head_neck

Part of the Otolaryngology Commons

\section{Recommended Citation}

Doty, R., Kisat, M., Tourbier, I. (2008). Estrogen replacement therapy induces functional asymmetry on an odor memory/ discrimination test. Brain Research, 1214, 35-39.

Available at: https://ecommons.aku.edu/pakistan_fhs_mc_surg_otolaryngol_head_neck/26 


\title{
Research Report
}

\section{Estrogen replacement therapy induces functional asymmetry on an odor memory/discrimination test}

\author{
Richard L. Doty ${ }^{a, *}$, Mehreen Kisat ${ }^{b}$, Isabelle Tourbier ${ }^{a}$ \\ ${ }^{a}$ Smell and Taste Center, Department of Otorhinolaryngology: Head and Neck Surgery, University of Pennsylvania School of Medicine, USA \\ ${ }^{\mathrm{b}}$ Aga Khan University Hospital, Karachi, Pakistan
}

\section{A R T I C L E I N F O}

Article history:

Accepted 8 April 2008

Keywords:

Laterality

Odor memory

Odor discrimination

Estrogen

Hormone replacement therapy

\begin{abstract}
A B S T R A C T
The secondary afferents of the olfactory system largely project to the ipsilateral cortex without synapsing in the thalamus, making unilateral olfactory testing a useful probe of ipsilateral hemispheric activity. In light of evidence that lateralized performance on some perceptual tasks may be influenced by estrogen, we assessed left:right nostril differences in two measures of olfactory function in 14 post-menopausal women receiving estrogen replacement therapy (ERT) and 48 post-menopausal women receiving no such therapy. Relative to women not taking ERT, those receiving ERT exhibited better performance in the left nostril and poorer performance in the right nostril on an odor memory/discrimination test. Similar laterality effects were not observed for an odor detection threshold test employing phenyl ethyl alcohol. These results suggest that estrogen influences the lateralization of an odor memory/discrimination task and that hormone replacement therapy in the menopause may be an excellent paradigm for understanding lateralizing effects of hormones on some sensory processes.
\end{abstract}

(C) 2008 Elsevier B.V. All rights reserved.

\section{Introduction}

The olfactory system is unique among the senses in that most of its secondary afferents project ipsilaterally to cortical regions without first synapsing in the thalamus (Gloor, 1997). Given this pattern of projections, unilateral olfactory testing is a useful probe of the function of the ipsilateral hemisphere (Doty et al., 1997). Odors presented to the left nostril are more easily named than those presented to the right nostril, reflecting left-hemisphere language processes (Gordon and Sperry, 1969; Herz et al., 1999; Homewood and Stevenson, 2001). In patients with seasonal affective disorder, odor identification scores obtained from the right, but not left, side of the nose correlate negatively with 'typical' depression scores on the Hamilton Depression Rating Scale $(r=-0.56$, $p=0.006)$ (Postolache et al., 1999), likely reflecting depressionrelated right-hemisphere anomalies (Baxter et al., 1989; Bench et al., 1993).

Recently we demonstrated that scores on a short-term odor memory/discrimination test were higher on the left than on the right side of the nose of women, but not men (Doty and Kerr, 2005). Handedness was unrelated to the effect. While this task did not require subjects to name or identify odors, the odors were ones that conceivably could be recognized, suggesting that the sex-related functional asymmetry could reflect differential use of semantic encoding or recall cues by men and women. It is well established that women are more likely than men to employ semantic strategies in remembering odors and that odor recognition memory is enhanced when veridical or generated names are given to odors (Jehl

\footnotetext{
* Corresponding author. Smell and Taste Center, University of Pennsylvania Medical Center, 5 Ravdin Building, 3400 Spruce Street, Philadelphia, PA 19104, USA.

E-mail address: doty@mail.med.upenn.edu (R.L. Doty).
} 
et al., 1997; Larsson et al., 2003; Oberg et al., 2002). Odor information processing likely shares a number of the cortical resources used in left-hemisphere language processing (Lorig, 1999).

Lateralization of some perceptual tasks fluctuates with phases of the menstrual cycle (Altemus et al., 1989; Hausmann et al., 2002; Hausmann and Gunturkun, 2000; Heister et al., 1989; Hollander et al., 2005; Mead and Hampson, 1996; Rode et al., 1995). For example, a greater left-hemisphere advantage has been found for a verbal dichotic listening task during the midluteal phase, when estrogens and progesterone are elevated, than during the menses, when such hormones are at a nadir. In contrast, a right-hemisphere advantage has been noted during the midluteal phase for a musical chord dichotic recognition listening task known to tap right-hemisphere neural processes (Sanders and Wenmoth, 1998). In the case of olfaction, $n$-butanol thresholds have been reported to be lower midcycle on the left than on the right side of the nose, whereas during menses the reverse is the case, again suggesting that hemispheric function is influenced by ovarian steroids, most notably estrogens (Purdon et al., 2001).

In this study we determined whether post-menopausal estrogen replacement therapy (ERT) alters the lateralization of scores on a short-term odor memory/discrimination test and an odor detection threshold test. We hypothesized that women on ERT would exhibit relatively better left nostril performance on the odor memory/discrimination test, but not on the odor detection threshold test, under the expectation that the memory/discrimination task would be more likely to engage left-hemisphere semantic processes than the detection task. If estrogen alters such lateralization, then unilateral olfactory testing may be a unique probe of the influences of estrogen on the two sides of the brain.

\section{Results}

\subsection{Odor memory/discrimination test}

Since no delay interval effects were present, as has been noted in other studies (Choudhury et al., 2003), the data were combined across delay intervals. The OMT scores were initially subjected to a nose side (left, right) by side of nose tested first (left, right) by hormone group (ERT, no ERT) analysis of covariance (ANCOVA), with repeated measures on nose side and the covariates of age, handedness score, left:right differences in PEA detection threshold, and left:right differences in nasal cross-sectional area or volume. ${ }^{1}$ The covariates were included to control for any potential contribution these measures may have had on the dependent measure. The only significant factor was a nose side by hormone group interaction $[F(1,54)=7.71, p=0.008]$, reflecting the fact that the ERT group exhibited better performance on the left side of the nose

\footnotetext{
${ }^{1}$ Separate analyses were performed using left:right differences in nasal volume and minimum cross-sectional area. In no case was these measures statistically significant and the overall findings were essentially the same. Hence, the statistical details are presented only for the analyses that used the left:right volume differences as a covariate.
}

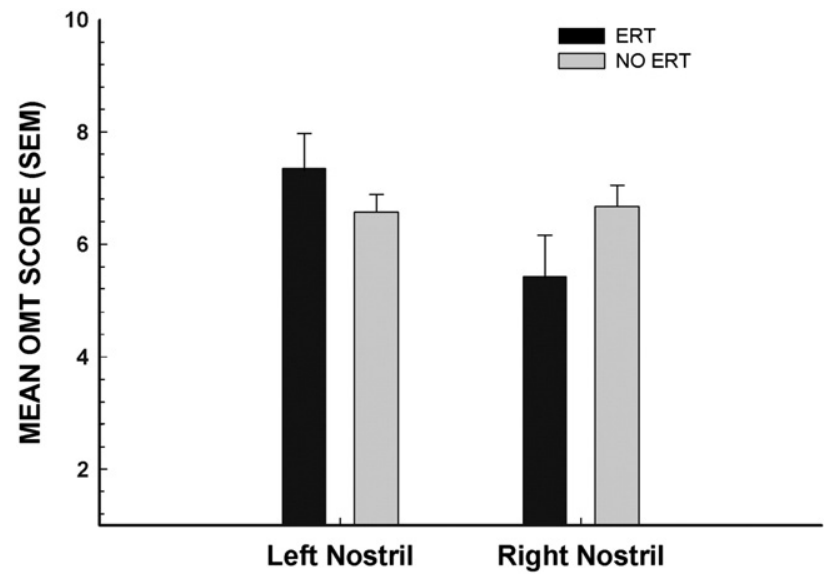

Fig. 1 - Mean ( \pm SEM) odor memory/discrimination test scores as a function of nose side and estrogen replacement therapy (ERT) group. See text for details.

and poorer performance on the right side of the nose, whereas the no-ERT group performed similarly on the two sides of the nose (Fig. 1). Post-hoc comparisons revealed a significant difference only between the left- and right-side performances of the ERT group $[t(13)=2.98, p=0.011]$.

\subsection{Phenyl ethyl alcohol (PEA) odor detection threshold test}

The PEA detection threshold values were subjected to the same ANCOVAs as noted above with the exception that the left:right PEA threshold difference score was omitted as a covariate. No significant interaction between hormone group and side of nose was observed $[F(1,55)=0.029, p=0.866]$ and no other factors or interactions were significant at the $0.05 \alpha$ level.

\section{Discussion}

The present study demonstrates that ERT alters left:right nostril performance on a short-term odor memory/discrimination test, but not on an odor detection threshold test employing the rose-like odorant phenyl ethyl alcohol. Specifically, women receiving ERT exhibited enhanced OMT performance on the left side of the nose and depressed OMT performance on the right side of the nose. This phenomenon was unrelated to handedness, age, PEA detection threshold sensitivity, or left:right differences in either nasal volume or minimum cross-sectional area, as determined from acoustic rhinometry. These findings are congruent with studies reporting that female reproductive hormones influence the lateralization of hemispheric cognitive processing (e.g., Kimura, 1996) and that some forms of memory may be facilitated in post-menopausal women by hormone replacement therapy (Maki et al., 2001). They also are in agreement with the concept that hormone-related alterations in olfactory function are not due to alterations in nasal engorgement (Caruso et al., 2001).

The physiological basis of ERT's influence on the laterality of the OMT scores is unknown. Estrogen's task-specific effects in humans appear similar to those observed in rodents. Thus, 
estrogens enhance performance in rats and humans on some types of seemingly related memory tasks (e.g., working memory, verbal memory) and impair performance on others (e.g., reference memory, spatial memory) (Dohanich, 2002). Presumably such influences reflect common neural processes dependent upon one or both of the known estrogen receptors $(E R)$, namely $E R \alpha$ and ER $\beta$. In female mice, hippocampaldependent spatial memory performance is depressed by estrogen administration, a phenomenon absent in mice lacking a functional copy of the ER $\alpha$ gene (Fugger et al., 1998). In male rats, $17 \beta$-estradiol, but not the nonaromatizable androgen $5 \alpha$-dihydrotesterone, significantly reverses the volume decrement of the left, but not right, posterodorsal nucleus of the medial amygdala (MePD) induced by castration (Cooke et al., 2003). The MePD has a high density of both ER $\alpha$ and ER $\beta$ receptors and plays a key role in integrating olfactory and neuroendocrine information (King, 2006). However, the mechanisms responsible for these effects are unlikely due to a simple left:right difference in estrogen receptor distribution, since the relative amount of ER mRNA-expression appears not to differ on the two sides of the brain (Shughrue and Merchenthaler, 2001).

In contrast to the only other study outside of our laboratory to report lateralized hormonal influences on olfaction (Purdon et al., 2001), we found no lateralizing effect of estrogen on the olfactory threshold. However, these two studies differ on a number of counts. First, our subjects were older (age-related decreases in the asymmetry of some neurotransmitters have been reported; Vernaleken et al., 2007). Second, we employed PEA rather than $n$-butanol which, unlike PEA, has more of a propensity to stimulate CN V (Doty et al., 1978). Third, based on odor quality, these two stimuli may differentially influence the left and right hemispheres (Henkin and Levy, 2001). Fourth, we compared post-menopausal women receiving and not receiving ERT, whereas Purdon et al. compared function during the menses to that during the midluteal phase of the menstrual cycle when estrogen and progesterone are both elevated. Chronic long-term ERT represents a very different hormonal milieu than shorter-term natural hormonal fluctuations during the menstrual cycle. Aside from hormonal differences, it is conceivable that the differences noted by Purdon et al. reflect menstrual cycle-related processes not directly involving ovarian steroids, as has been suggested for some measures of olfactory sensitivity (Doty et al., 1981). Finally, Purdon et al. employed a single point in an ascending concentration series to assess threshold, whereas we averaged multiple points to achieve this end. To determine if this might explain the discrepant findings, we re-evaluated our threshold data using only the first reversal as the threshold estimate. No significant effect of ERT was found, making this explanation appear unlikely.

In summary, the present study suggests that ERT alters the laterality of performance on an odor memory/discrimination task in post-menopausal women, adding to other evidence that female hormones may have differential influences on the two sides of the brain (e.g., Kimura 1996). This phenomenon seems to be independent of any influence on olfactory sensitivity, per se, as measured by a single staircase detection threshold test employing PEA. Since only women taking unopposed estrogen were evaluated in this research, and our sample size of such women was relatively small, additional studies are needed to validate our findings and to determine whether they generalize to other types of hormone replacement therapy used in the post-menopausal period. This is particularly relevant in light of evidence suggesting that combination estrogen-progesterone therapy, unlike ERT alone, may decrease verbal fluency scores and accelerate cognitive decline (Flor et al., 2002; Grady et al., 2002; Rice et al., 2000).

\section{Experimental procedures}

\subsection{Subjects}

Sixty-two women served as subjects [mean (SD) age $=58.98$ (9.14)]. They were selected from a larger group of individuals who attended our clinic but who evidenced relatively normal age-related smell function, as defined by scores on the University of Pennsylvania Smell Identification Test (UPSIT) [median (range)=34.00 (27-40)] (Doty et al., 1984). All were post-menopausal by self-report. Scores on the Mini-Mental State Examination (MMSE) revealed no meaningful cognitive dysfunction [mean (SD)=29.13 (1.15); median=29.5].

The subjects were divided into those taking $(n=14)$ and those not taking $(n=48)$ ERT. These two groups did not differ significantly from one another on the basis of age [respective means (SDs)=61.07 (7.94) and 58.69 (9.20)], Briggs and Nebes Handendess Score [17.21 (16.65) and 17.06 (12.96), or UPSIT score [33.21 (4.39) and 33.77 (3.10)] (all ps=0.38). Two of the ERT subjects and five of the non-ERT subjects were classified according to the Briggs and Nebes criteria as being left handed. All provided written informed consent regarding the use of their data, in accord with the requirements of the University's Office of Regulatory Affairs.

\subsection{Odor memory/discrimination assessment}

Short-term odor memory/discrimination was assessed using a 12-item four-alternative forced-choice test (Odor Memory Test $^{\mathrm{TM}}$, Sensonics, Inc., Haddon Heights, NJ). This test employs 10-, 30-, and 60-second delay intervals between the presentation of a target odorant and the first of four successively presented odorants from which the target is selected (Doty, 2003). The target odorant is initially released by scratching an odorized label that is then presented to the subject for sampling. After a given delay interval, four subsequent odorants (the target and three foils) are similarly released and presented at $\sim$ five second intervals. The subject's task is to report which odor in the odor response set is the same as the target stimulus. During the delay interval, the subject counts aloud backwards by threes from 280 to minimize verbal rehearsal. The presentation order of the stimuli is counterbalanced such that (a) all target odorants occur an equal number of times at each delay interval, (b) each target odorant is represented at a given delay interval once in each of the four possible response positions (i.e., a, b, c, and d), and (c) all four odorants are presented in the first, second, and third segments of the test. The test-retest reliability of the OMT is $\sim 0.75$. 


\subsection{Odor detection threshold assessment}

A two-alternative forced-choice single staircase procedure was used to establish detection threshold values for the roselike smelling odorant phenyl ethyl alcohol (PEA). The staircase began at the -6.00 log concentration step of a half-log step (vol/vol) dilution series extending from $-10.00 \mathrm{log}$ to $-2.00 \mathrm{log}$ concentration. The stimulus concentration was increased in full log steps until correct detection occurred on five sets of consecutive trials at a given concentration. If an incorrect response was given on any trial, the staircase was moved upward one full log step. When a correct response was made on all five trials, the staircase was reversed and subsequently moved up or down in 0.50 log increments or decrements, depending upon the subject's performance on two pairs of trials at each concentration step. The geometric mean of four staircase reversal points following the third staircase reversal was used as the threshold measure. The test-retest reliability of this instrument is $>0.80$ (Doty et al., 1995).

\subsection{Measurement of nasal volume and minimum cross-sectional area}

The volume and minimum cross-sectional area of each side of the nose were assessed using an Eccovision ${ }^{\circledR}$ Acoustic Rhinometer (Pembroke, MA). This device acts similar to sonar or radar, projecting sound waves in the form of a series of clicks into each side of the nose and measuring their reflections via a microphone to assess dimensional elements of the nasal cavity. The silastic nosepiece that connects the wave tube to the nose was individually fitted for each subject. The wave tube was positioned near the midline $\sim 45^{\circ}$ off vertical and adjusted slightly to produce a stable trace (Moberg et al., 2004).

\subsection{Procedure}

The dependent measures of this study, i.e., the OMT and the PEA detection threshold test, were each administered to the left and right sides of the nose in a counterbalanced order. The naris opposite to the one being tested was occluded using a piece of Microfoam ${ }^{\mathrm{TM}}$ tape (3M Corporation, Minneapolis, MN) fitted carefully over the ala nasi and nasal sill, a procedure that does not deform either nasal opening. The subjects were asked to breathe in through the nose and out through the mouth during testing. The nasal volumes and minimal crosssectional areas were similarly determined using the acoustic rhinometer, although in this case the subject was required to hold his or her breath and the opposite nasal chamber was not occluded. The UPSIT, which was performed bilaterally, was administered after the OMT to preclude any possible sensitization of the subjects to odor names. In approximately half of the cases acoustic rhinometry was performed prior to the olfactory testing and half after the olfactory testing.

\section{Acknowledgments}

This article was supported by the National Institute of Aging Grant RO1 AG17496-5 to Richard L. Doty. Mehreen Kisat was a visiting summer scholar and was supported by funds from the
Aga Khan University Hospital, Karachi, Pakistan. Disclosure: Richard L. Doty is a major shareholder in Sensonics, Inc., the manufacturer of the olfactory tests employed in this study.

\section{R E F E R E N C E S}

Altemus, M., Wexler, B.E., Boulis, N., 1989. Changes in perceptual asymmetry with the menstrual-cycle. Neuropsychologia 27, 233-240.

Baxter, L.R., Schwartz, J.M., Phelps, M.E., Mazziotta, J.C., Guze, B.H., Selin, C.E., Gerner, R.H., Sumida, R.M., 1989. Reduction of prefrontal cortex glucose-metabolism common to 3 types of depression. Arch. Gen. Psychiat. 46, 243-250.

Bench, C.J., Friston, K.J., Brown, R.G., Frackowiak, R.S.J., Dolan, R.J., 1993. Regional cerebral blood-flow in depression measured by positron emission tomography - the relationship with clinical dimensions. Psychol. Med. 23, 579-590.

Caruso, S., Grillo, C., Agnello, C., Maiolino, L., Intelisano, G., Serra, A., 2001. A prospective study evidencing rhinomanometric and olfactometric outcomes in women taking oral contraceptives. Hum. Reprod. 16, 2288-2294.

Choudhury, E.S., Moberg, P., Doty, R.L., 2003. Influences of age and sex on a microencapsulated odor memory test. Chem. Senses 28, 799-805.

Cooke, B.M., Breedlove, S.M., Jordan, C.L., 2003. Both estrogen receptors and androgen receptors contribute to testosterone-induced changes in the morphology of the medial amygdala and sexual arousal in male rats. Horm. Behav. 43, 336-346.

Dohanich, G., 2002. Gonadal steroids, learning, and memory. In: Pfaff, D.W., Arnold, A.P., Etgen, A.M., Fahrbach, S.E., Rubin, R.T. (Eds.), Horm., Brain and Behav. Academic Press, San Diego, pp. 265-327.

Doty, R.L., Brugger, W.E., Jurs, P.C., Orndorff, M.A., Snyder, P.J., Lowry, L.D., 1978. Intranasal trigeminal stimulation from odorous volatiles: psychometric responses from anosmic and normal humans. Physiol. Behav. 20, 175-185.

Doty, R.L., Snyder, P.J., Huggins, G.R., Lowry, L.D., 1981. Endocrine, cardiovascular, and psychological correlated of olfactory sensitivity changes during the human menstrual cycle. J. Comp. Physiol. Psychol. 95, 45-60.

Doty, R.L., Shaman, P., Dann, M., 1984. Development of the University of Pennsylvania Smell Identification Test: a standardized microencapsulated test of olfactory function. Physiol. Behav. 32, 489-502.

Doty, R.L., McKeown, D.A., Lee, W.W., Shaman, P., 1995. A study of the test-retest reliability of ten olfactory tests. Chem. Senses 20, 645-656.

Doty, R.L., Bromley, S.M., Moberg, P.J., Hummel, T., 1997. Laterality in human nasal chemoreception. In: Christman, S. (Ed.), Cerebral Asymmetries in Sensory and Perceptual Processing. North Holland Publishing Co., Amsterdam, pp. 497-542.

Doty, R.L., 2003. The Odor Memory Test ${ }^{\mathrm{TM}}$ Administration Manual, 2nd Edition. Sensonics, Inc, Haddon Hts., NJ.

Doty, R.L., Kerr, K.L., 2005. Episodic odor memory: influences of handedness, sex, and side of nose. Neuropsychologia 43, 1749-1753.

Flor, H., Birbaumer, N., Hermann, C., Ziegler, S., Patrick, C.J., 2002. Aversive Pavlovian conditioning in psychopaths: peripheral and central correlates. Psychophysiology 39, 505-518.

Fugger, H.N., Cunningham, S.G., Rissman, E.F., Foster, T.C., 1998. Sex differences in the activational effect of ER alpha on spatial learning. Horm. Behav. 34, 163-170.

Gloor, P., 1997. The Temporal Lobe and the Limbic System. Oxford University Press, New York. 
Gordon, H.W., Sperry, R.W., 1969. Lateralization of olfactory perception in the surgically separated hemispheres of man. Neuropsychologia 7, 111-120.

Grady, D., Yaffe, K., Kristof, M., Lin, F., Richards, C., Barrett-Connor, E., 2002. Effect of postmenopausal hormone therapy on cognitive function: the heart and estrogen/progestin replacement study. Amer. J. Med. 113, 543-548.

Hausmann, M., Gunturkun, O., 2000. Steroid fluctuations modify functional cerebral asymmetries: the hypothesis of progesterone-mediated interhemispheric decoupling. Neuropsychologia 38, 1362-1374.

Hausmann, M., Becker, C., Gather, U., Gunturkun, O., 2002. Functional cerebral asymmetries during the menstrual cycle: a cross-sectional and longitudinal analysis. Neuropsychologia $40,808-816$.

Heister, G., Landis, T., Regard, M., Schroeder-Heister, P., 1989. Shift of functional cerebral asymmetry during the menstrual cycle. Neuropsychologia 27, 871-880.

Henkin, R.I., Levy, L.M., 2001. Lateralization of brain activation to imagination and smell of odors using functional magnetic resonance imaging (fMRI): left hemispheric localization of pleasant and right hemispheric localization of unpleasant odors. J. Comp. Assist Tomogr. 25, 493-514.

Herz, R.S., McCall, C., Cahill, L., 1999. Hemispheric lateralization in the processing of odor pleasantness versus odor names. Chem Senses 24, 691-695.

Hollander, A., Hausmann, M., Hamm, J.P., Corballis, M.C., 2005. Sex hormonal modulation of hemispheric asymmetries in the attentional blink. J. Internat. Neuropsychol. Soc. 11, 263-272.

Homewood, J., Stevenson, R.J., 2001. Differences in naming accuracy of odors presented to the left and right nostrils. Biol. Psychol. 58, 65-73.

Jehl, C., Royet, J.P., Holley, A., 1997. Role of verbal encoding in short- and long-term odor recognition. Percept. Psychophys. 59, 100-110.

Kimura, D., 1996. Sex, sexual orientation and sex hormones influence human cognitive function. Curr. Opin. Neurobiol. 6, 259-263.

King, B.M., 2006. Amygdaloid lesion-induced obesity: relation to sexual behavior, olfaction, and the ventromedial hypothalamus. Amer. J. Physiol. Reg. Integ. Comp. Physiol. 291, R1201-R1214.
Larsson, M., Lovden, M., Nilsson, L.G., 2003. Sex differences in recollective experience for olfactory and verbal information. Acta Psychol. 112, 89-103.

Lorig, T.S., 1999. On the similarity of odor and language perception. Neurosci. Biobehav. Rev. 23, 391-398.

Maki, P.M., Zonderman, A.B., Resnick, S.M., 2001. Enhanced verbal memory in nondemented elderly women receiving hormone-replacement therapy. Am. J. Psychiatry 158, 227-233.

Mead, L.A., Hampson, E., 1996. Asymmetric effects of ovarian hormones on hemispheric activity: evidence from dichotic and tachistoscopic tests. Neuropsychology 10, 578-587.

Moberg, P.J., Roalf, D.R., Gur, R.E., Turetsky, B.I., 2004. Smaller nasal volumes as stigmata of aberrant neurodevelopment in schizophrenia. Am. J. Psychiatry 161, 2314-2316.

Oberg, C., Larsson, M., Backman, L., 2002. Differential sex effects in olfactory functioning: the role of verbal processing. J. Internat Neuropsychol. Soc. 8, 691-698.

Postolache, T.T., Doty, R.L., Wehr, T.A., Jimma, L.A., Han, L., Turner, E.H., Matthews, J.R., Neumeister, A., No, C., Kroger, H., Bruder, G.E., Rosenthal, N.E., 1999. Monorhinal odor identification and depression scores in patients with seasonal affective disorder. J. Affect. Dis. 56, 27-35.

Purdon, S.E., Klein, S., Flor-Henry, P., 2001. Menstrual effects on asymmetrical olfactory acuity. J. Internat Neuropsychol. Soc. 7, 703-709.

Rice, M.M., Graves, A.B., McCurry, S.M., Gibbons, L.E., Bowen, J.D., McCormick, W.C., Larson, E.B., 2000. Postmenopausal estrogen and estrogen-progestin use and 2-year rate of cognitive change in a cohort of older Japanese American women: the Kame Project. Arch. Intern. Med. 160, 1641-1649.

Rode, C., Wagner, M., Gunturkun, O., 1995. Menstrual cycle affects functional cerebral asymmetries. Neuropsychologia 33, 855-865.

Sanders, G., Wenmoth, D., 1998. Verbal and music dichotic listening tasks reveal variations in functional cerebral asymmetry across the menstrual cycle that are phase and task dependent. Neuropsychologia 36, 869-874.

Shughrue, P.J., Merchenthaler, I., 2001. Distribution of estrogen receptor beta immunoreactivity in the rat central nervous system. J. Comp. Neurol. 436, 64-81 Jul 16.

Vernaleken, I., Weibrich, C., Siessmeier, T., Buchholz, H.G., Rosch, F., Heinz, A., Cumming, P., Stoeter, P., Bartenstein, P., Grunder, G., 2007. Asymmetry in dopamine D-2/3 receptors of caudate nucleus is lost with age. NeuroImage $34,870-878$. 